\title{
Inductive logic programming for gene regulation prediction
}

\author{
Sebastian Fröhler · Stefan Kramer
}

Received: 10 October 2007 / Accepted: 11 October 2007 / Published online: 9 November 2007

Springer Science+Business Media, LLC 2007

\begin{abstract}
We present a systems biology application of ILP, where the goal is to predict the regulation of a gene under a certain condition from binding site information, the state of regulators, and additional information. In the experiments, the boosted Tilde model is on par with the original model by Middendorf et al. based on alternating decision trees (ADTrees), given the same information. Adding functional categorizations and protein-protein interactions, however, it is possible to improve the performance substantially. We believe that decoding the regulation mechanisms of genes is an exciting new application of learning in logic, requiring data integration from various sources and potentially contributing to a better understanding on a system level.
\end{abstract}

Keywords Inductive logic programming · Relational learning · Gene regulation · Gene expression · Systems biology

\section{Introduction}

The emerging discipline of systems biology aims at the precise mathematical modeling of cells, tissues, organs, organisms and ecosystems on a system-wide level. Most of the current work focuses on Petri net or ODE models of particular pathways of metabolic or regulatory networks. In this paper, we propose a systems biology application of inductive logic programming (ILP). In contrast to the mainstream of systems biology research, our goal is to integrate all available information about a simple eukaryotic model organism (yeast) and model an important aspect, that of gene regulation, statistically and comprehensively. We address the following question: Is it possible to predict gene expression from the state of known

Editors: Stephen Muggleton, Ramon Otero, Simon Colton.

S. Fröhler · S. Kramer ( $\varangle)$

Institut für Informatik, Technische Universität München, Boltzmannstr. 3, 85748 Garching bei

München, Germany

e-mail: kramer@in.tum.de

S. Fröhler

e-mail: s@froehler.info 
or putative regulators, binding site information, and additional information using ILP? Our model can be applied to a given regulatory region and the state of a set of regulators, and predicts whether the gene is up- or down-regulated. A gene is up- or down-regulated if the expression level is changed with respect to a reference condition. This is typically determined by a comparison between a stimulated (experimental) and a non-stimulated (reference) state. Genes whose expression levels increase under a stimulated condition are called up-regulated, analogously, they are called down-regulated.

Our starting point is the first predictive model of gene regulation proposed by Middendorf et al. (2004). As we will argue, the task of gene regulation prediction is ideal for ILP, as data from various sources need to be integrated to build a strong predictive model. A specific advantage of ILP in this application area is the flexibility to adjust the bias to complex data and the inherent extensibility, that is, the possibility to incorporate any information that might be useful for the task at hand.

This paper is organized as follows: In Sect. 2, we explain the biological background of eukaryotic gene regulation as needed for the purpose of this paper. In Sect. 3, the type and origin of the data as well as their representation is presented. Section 4, the main part of the paper, discusses the results from a quantitative and qualitative point of view. Finally, we draw our conclusions and sketch directions of further work in Sect. 5.

\section{Background on eukaryotic gene regulation}

In the following section, we will give a short overview of eukaryotic gene regulation. Generally, genes are not regulated independently of each other, but as functional groups/modules. Functional modules are sets of genes or gene products that are part of the same biological process or are involved in the same biological function. This may be because the gene products interact with each other in protein-protein interactions, or because they are part of the same reaction pathway.

The components of a functional module depend on each other. The deletion of just one component from a functional module can render the complete module non-functional. An example could be the deletion of one enzyme in a non-redundant metabolic pathway which leads to an interruption of this metabolic pathway. So, the genes of a functional module need to be regulated jointly to ensure the functionality of the respective functional module.

Whereas in prokaryotes, co-regulation of genes is typically performed by encoding the respective genes in the same operon, eukaryotic gene regulation is much more complicated. Since in eukaryotes each gene has its own regulatory region, there is no need to encode co-regulated genes in close proximity to each other. In fact, co-regulated genes can be distributed across the whole genome.

We will now inspect the structure of a eukaryotic gene and relate this structure to the concept of functional modules (see Fig. 1). A eukaryotic gene can be roughly divided into two parts: The coding region and the regulatory region. The coding region contains the "content" of the gene which is the encoded gene product. This gene product is encoded in a number of exons which are divided by introns which in turn allow the mechanism of alternative splicing leading to different variants of the gene product.

The regulatory region contains several elements which are important for the transcription of a gene: The binding site for the basal transcription machinery in the vicinity of the 
a)

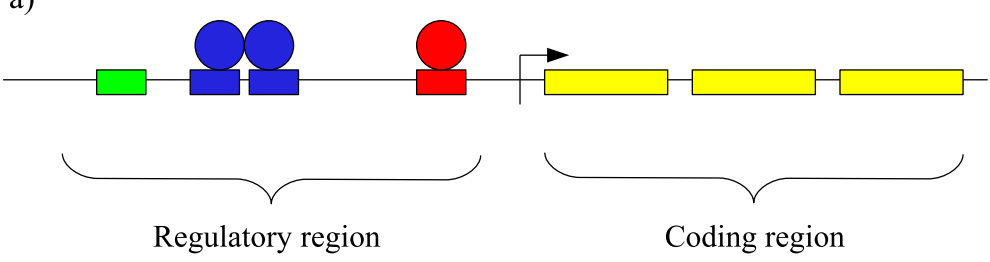

b)

Module 1

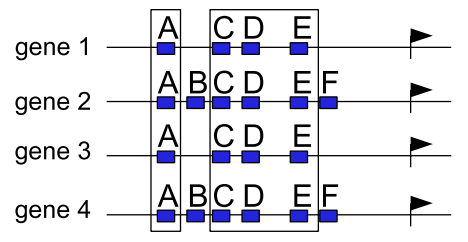

Module 3

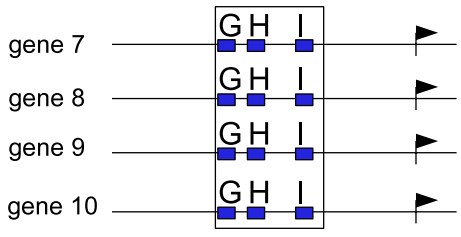

Module 2

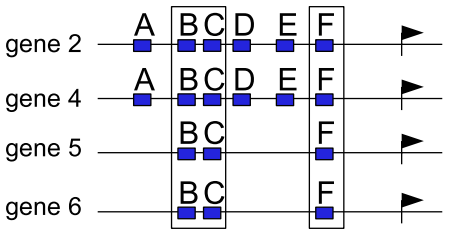

Fig. 1 a Structure of a eukaryotic gene. The regulatory region contains transcription factor binding sites (boxes), some of these binding sites are bound by transcription factors (circles). b Three regulatory modules. Module 1 and module 2 share the transcription factor binding site ' $\mathrm{C}$ ', module 3 does not share any binding sites. Genes 2 and 4 belong to both: module 1 and module 2

transcription start site together with binding sites for general transcription factors ${ }^{1}$ and a set of transcription factor binding sites for regulatory transcription factors ${ }^{2}$.

More generally, regulators are gene products influencing (regulating) the expression level of other gene products. This can be done by binding to a binding site located upstream of the regulated gene or by modifying the activity/localization of another regulator that can either bind to an upstream binding site of the regulated gene or in turn modify the activity/localization of another regulator as part of a signaling/regulatory chain/cascade. Regulators can be either transcription factors, signaling molecules, or protein kinases.

A gene is part of a functional module if the regulatory transcription factor binding sites of that module can be found in the regulatory region of the respective gene. So the expression of each functional module is controlled by a specific set of regulatory transcription factor binding sites in the regulatory region of all genes of that module. If a gene belongs to more than one functional module, its regulatory region also contains the sets of regulatory transcription factor binding sites of all other functional modules of that gene (see Fig. 1).

\footnotetext{
${ }^{1}$ General transcription factors are required for the process of transcription of all genes.

${ }^{2}$ Regulatory transcription factors control the process of transcription of a specific gene or a functional module in an environment dependent-manner.
} 
It is important to mention that different functional modules may have common subsets of regulatory transcription factor binding sites. Also, not all regulatory transcription factor binding sites of a module have to be bound by transcription factors under each condition the module is required - a subset of all binding sites of a module can be bound under a specific condition. This can be the case if, for instance, two or more alternative binding sites for a specific class of activators ${ }^{3}$ can be found in the regulatory region of the respective genes and the binding of one of these activators to the respective binding site is sufficient to activate transcription.

On a molecular level, functional modules can be characterized by a set of transcription factor binding sites. On a higher level, these modules can be characterized by functional categories, as in the FunCat (Ruepp et al. 2004) database. For instance, a functional module is given by FunCat category 02.10, which contains genes of the citrate cycle. The set of regulatory transcription factor binding sites of a functional module characterizes the regulatory program of that module. The evaluation of the program depends on the set of active transcription factors under a specific condition. Generally, a regulatory transcription factor binding site is specifically bound by a transcription factor under a specific condition. A transcription factor can either modify the chromatin structure ${ }^{4}$ or modify the activity/stability of the basal transcription machinery near the transcription start site. The latter effect finally can lead to an increased or decreased transcription of a gene under a specific condition, whereas the first effect can disable transcription of a whole chromosomal region. ${ }^{5}$

The environment of the cell influences the transcriptional program of the cell. So the gene regulation program encoded in the regulatory region of a gene together with the environmental conditions influencing the presence and activity of transcription factors together are responsible for a specific phenotype of a cell. According to Latchman (2005), the major targets for gene regulation are the initiation of transcription together with transcription elongation. Another important target is post-transcriptional and post-translational control of gene products in an environment-dependent manner. In this work, we will focus on transcription initiation and elongation, which can be monitored using microarrays. Like Segal et al. (2003), we make the assumption that the behavior of most regulators and signaling molecules themselves are controlled via their expression levels.

\section{Data and representation}

\subsection{Definition of learning task}

Studying gene regulation mechanisms in simple model organisms has emerged as an important problem in bioinformatics. In most cases, the analysis is restricted to descriptive methods. The first predictive model for gene regulation was proposed by Middendorf et al. (2004). In this approach, the presence of a transcription factor binding site in the regulatory region of a gene together with the expression state of regulators is used to predict the regulatory state of genes. A similar approach is taken by Ong et al. (2006), where the time course of gene expression is modeled in an ILP setting. ${ }^{6}$

\footnotetext{
${ }^{3} \mathrm{An}$ activator is a regulatory transcription factor that is able to activate transcription.

${ }^{4}$ The chromatin structure influences the expression of genes of a specific chromosomal region. These genes do not necessarily have to be part of the same functional module - this is a higher level of gene regulation.

${ }^{5}$ This is achieved by a tight packing of DNA, making it inaccessible for the transcription machinery.

${ }^{6}$ The differences and commonalities will be discussed in the conclusion section.
} 
In this work, we develop a model able to predict the regulatory state of a gene under a specific experimental condition given some information about that gene and certain aspects of the experimental condition. Beyond the datasets already used by Middendorf et al., we integrate information about protein-protein interactions and functional categorizations of genes. The ILP approach is particularly well-suited for the task at hand, since it offers the opportunity to incorporate biological data as needed. Incorporating more knowledge relevant for gene regulation allows to model the process of gene regulation more completely and to obtain better and more detailed descriptions of gene regulatory mechanisms under a specific set of experimental conditions. The resulting model is required to be complex enough to capture interrelationships relevant for gene regulation, yet simple enough for the extraction and interpretation of important features.

\subsection{Data retrieval and preprocessing}

We will now introduce the different kinds of data we use and the preprocessing for each of these datasets.

\subsubsection{Gene expression data}

We downloaded the Gasch et al. (2000) environmental stress dataset containing gene expression data for about 6,000 Saccharomyces cerevisiae genes under 173 experimental conditions (as of June 2001). These conditions comprise diverse environmental stress situations like temperature shocks, hydrogen peroxide, hyper- and hypo-osmotic shock, amino acid starvation, nitrogen source depletion and progression into stationary phase. Roughly 900 genes show a similar drastic response to almost all of these environmental changes. These and other stress-responding genes are the targets for our analysis.

Each value in the gene expression matrix represents a background-corrected, columncentered ${ }^{7} \log _{2}$ gene expression value for a specific gene under a specific experimental condition. Bad spots ${ }^{8}$ and spots with very low intensities were removed by the experimenters during array post-processing (Audrey Gasch, personal communication). More details on the methods used for generating the environmental stress dataset can be found in the paper by Gasch et al. (2000).

Because the post-processing as described by Gasch et al. seemed very reasonable, no further post-processing of the dataset was performed. All entries of the gene expression dataset were discretized using an empirical noise cut-off. This was calculated from the unstimulated reference time zero noise replicates that are contained in the data. More precisely, the cut-off is $\log _{2}>= \pm 1.2$, meaning that $95 \%$ of all expression values lie between -1.2 and +1.2 in the noise replicates (see Fig. 2). This discretization of the expression dataset is analogous to the discretization performed by Middendorf et al. (2004). To test the effect of this discretization, we will also present an experiment on undiscretized data in Sect. 4.

\subsubsection{Transcription factor binding site data}

We obtained the transcription factor binding sites for 6,000 Saccharomyces cerevisiae genes from Middendorf et al. (2004) (as of March 2004). These binding sites were found by scanning the 500 bp upstream sequence of each Saccharomyces cerevisiae gene for the occurrence of binding sites using the Patch tool of the TRANSFAC database (Wingender et al.

\footnotetext{
${ }^{7}$ One column of the gene expression matrix represents one experimental condition.

${ }^{8} \mathrm{~A}$ spot on the microarray represents the ratio of two experiments: experiment condition vs. control condition.
} 
Fig. 2 Distribution of expression values in the unstimulated noise replicates. $95 \%$ of all expression values can be found between -1.2 and +1.2
Distribution of noise in the three null replicates

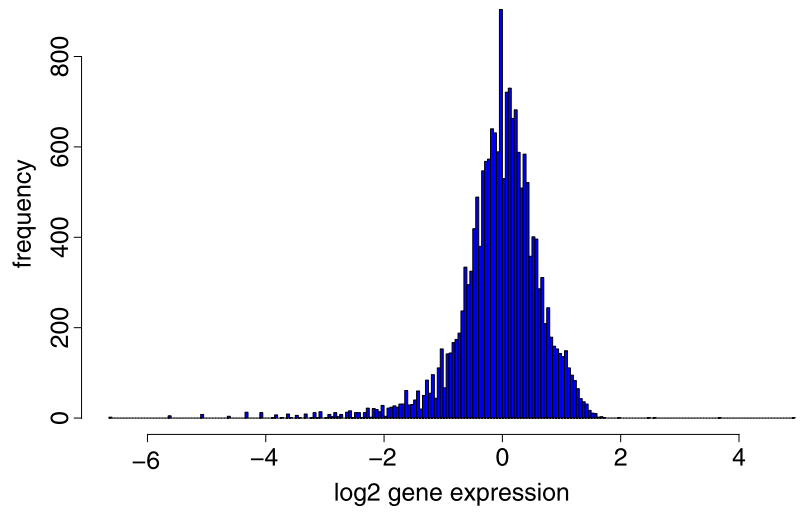

1996). The Patch tool is a search tool to identify transcription factor binding sites in the regulatory regions of a set of genes. Each binding site found represents a match of one of the weight matrices stored in TRANSFAC scoring above a certain threshold. Default parameters of the Patch tool were used. As each entry in the matrix represents a count of the occurrence of a binding site in a gene, we discretized these values by only considering if a transcription factor binding site can be found in the upstream sequence of a gene (value 1) or not (value 0 ).

\subsubsection{Protein-protein interaction data}

We obtained all protein-protein interaction (PPI) data for Saccharomyces cerevisiae from MIPS (Mewes et al. 1997). These data contain all known physical and genetic interactions of proteins from Saccharomyces cerevisiae (as of December 22, 2005) in a binary form. Protein-protein interactions are represented as pairs of interacting proteins in this dataset. PPIs may result from high-throughput experiments, or, more reliably, manual annotation.

\subsubsection{Functional category data}

We retrieved the functional catalogue FunCat (Ruepp et al. 2004) from MIPS containing all annotated functional categories for Saccharomyces cerevisiae genes (as of November 14, 2005). FunCat represents functions from a general (e.g., FunCat 11: Protein Synthesis) to a quite specific level of detail (e.g., FunCat 11.02.03.04.01: transcriptional activator). Note that gene-products can be assigned to more than one FunCat term depending on the processes they are part of, and that FunCat focuses on the functional process a gene-product is part of and does not describe its molecular function to the atomic detail. Moreover, FunCat terms can be assigned manually as well as automatically (Ruepp et al. 2004). Functional category information has been shown to be useful for identifying co-regulated genes in a recent study by Allocco et al. (2004).

\subsection{Restrictions and assumptions}

Since noise and errors are inevitable in biological databases, a few assumptions and restrictions are in order, to keep our goal, predicting regulatory response of genes, feasible. 


\subsubsection{Discretization of gene expression values}

Gene expression data contains a significant level of noise. We therefore discretize gene expression data using an empirical noise cut-off estimated from the data as described above. So instead of using real-valued gene expression data, genes are discretized into three classes: down-regulated $(-1)$, no-change $(0)$ and up-regulated $(+1)$.

\subsubsection{Region for transcription factor binding sites search}

The region to search for transcription factor binding sites using TRANSFAC was limited to the $500 \mathrm{bp}$ upstream sequence of a gene, counted from the translation start site. ${ }^{9}$ There is a trade-off between the length of the region to scan for transcription factor binding sites and the fraction of false positives in the result. If the region is shorter, fewer false positives can be assumed, if it is longer, more false positives, but also more real binding sites can be discovered. According to van Helden et al. (1998), in yeast, regulatory elements are found almost exclusively upstream from the transcription start site. Additionally, most of the transcription factor binding sites in Saccharomyces cerevisiae (99\%) are located within the first $800 \mathrm{bp}$ upstream of the translation start site of a gene. We decided to use the same region to scan for binding sites as Middendorf et al. (2004) — 500 bp upstream of a gene.

\subsubsection{Occurrence of transcription factor binding sites}

To avoid overfitting, transcription factor binding sites are assumed to be either present or absent in the regulatory region of a particular gene. We do not take into account information about the number of transcription factor binding sites or the relative position of these sites to each other and to the transcription start site.

\subsubsection{Redundant transcription factor binding sites}

Several binding sites can occur jointly in the regulatory region of a gene. Here we define a redundant binding site as a site that has the same binding profile for the genes in the binding site matrix as another binding site. The binding site matrix is a $0 / 1$ matrix with the genes as the rows and their transcription factors as the columns. Since redundant binding sites provide the same information, it is safe to group them and keep only one representative. The names of all redundant binding sites of a group are kept for traceability.

\subsubsection{Representation of experimental conditions}

Experimental conditions are represented by the regulatory states of the transcription factors used in this work. Each condition corresponds to a set of discretized transcription factor gene expression values. Since we further assume that a transcription factor is only active if it is either up- or down-regulated, transcription factors that are assigned to state "no-change" under a specific condition are not regarded as predictive for that condition, since they do not change their expression state with respect to the reference condition. Thus, this transcription factor is not used as a predictive feature by our method under this condition.

\footnotetext{
${ }^{9}$ In eukaryotes, the transcription start site can hardly be predicted on the sole basis of the sequence (van Helden et al. 1998). Because of this, the beginning of the ORF (the translation start site) was chosen instead.
} 
Because some transcription factors are regulated by post-transcriptional or posttranslational mechanisms, these specific transcription factors are likely not to be used as predictive features for our method under any condition. Since we do not have high-throughput protein-activity data, we cannot monitor the behavior of these transcription factors and therefore we decided not to use those that are in a "no-change" state. As soon as protein activity data becomes available on a global scale (for the conditions studied), this piece of information could further improve predictive accuracy, because then the state of more potential regulators could be monitored. Note that this new data, when available, can be seamlessly integrated into the approach presented here.

\subsubsection{Target gene selection}

As Middendorf et al., we restrict ourself to a set of genes including 469 highly-variant genes with a standard deviation in expression over all experiments greater than 1.2, and 1,250 genes that occur in the 17 clusters identified by Gasch et al. (2000). In other words, we only keep genes that change over the experimental conditions and are not outliers. The overall list contains 1,411 distinct genes.

We only included genes from the binding site dataset with at least one transcription factor binding site found per gene. Since the main target for transcription regulation is the set of binding sites of a gene, this seems to be a reasonable choice.

\subsubsection{Regulator selection}

The set of regulators we use in this work consists of 13 highly-variant genes from the dataset that have a standard deviation greater than 1.2 across all experimental conditions, and 50 top-ranking regulator genes from the clusters identified by Segal et al. (2003). There are 53 regulators in the non-redundant set. A superset of 475 regulators did not change the results substantially (Fröhler 2006). Note that the 53 regulators are either transcription factors, protein kinases or signaling molecules and therefore can be regarded as biologically relevant regulators of transcription.

\subsection{Data representation and setup}

An overview of the defined predicates and relations can be found in Table 1. Information about the target genes is stored in the relation

$$
\text { gene (GeneID, ConditionID, ExpressionValue), }
$$

Table 1 Predicates and relations for diverse sources of data
Defined predicates

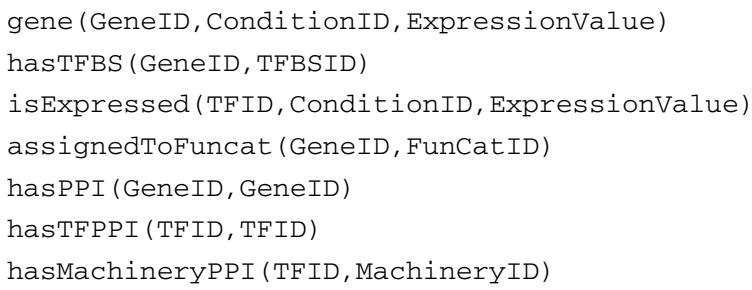


with the gene name, the condition identifier, and the discretized expression value of the gene under that condition as arguments. Binding site information is stored in

$$
\text { hasTFBS (GeneID, TFBSID), }
$$

with the name of the gene and a binding site identifier that can be found in the regulatory region of the gene. Information about the regulators is stored in the relation

$$
\text { isExpressed (TFID, ConditionID, ExpressionValue), }
$$

with the name of the regulator, a condition identifier, and a discretized expression value under that condition. It should be noted that the information from the three tables could be joined into a single propositional table with a lot of redundancy in the representation, but without loss of information or meaning. We will discuss this point in detail in the section on experimental results.

Functional category information per gene is stored in the relation assignedToFuncat (GeneID, FunCatID), with the gene name and the FunCat term assigned to the gene. This relation contains the FunCat term that was assigned to a gene and all of its parent terms as separate entries.

Protein-protein interaction data is stored in three different relations:

- hasPPI (GeneID, GeneID) containing PPIs between target genes,

- hasTFPPI (TFID, TFID) containing PPIs between regulators only, and

- hasMachineryPPI (TFID, MachineryID) containing protein-protein interactions between a regulator and a component of the transcription machinery (see also Table 1).

These predicates represent $m: n$ relationships. In the experiments, we will test all PPIs as input (i.e., also including potentially unreliable high-throughput data), and, separately, only PPIs from manual annotation.

The goal of the learning task is to learn the gene predicate in terms of all other predicates. In particular, the expression value is to be predicted given the gene identifier and the condition identifier. In order to achieve this goal, we restricted the language bias of the tool used, Tilde (Blockeel and Raedt 1998), such that all other information can only be added to a branch if it already contains an isExpressed literal. With this restriction, we want to ensure that all other (condition-independent) information can only be used in the context of a set of specific conditions.

\section{Experimental results and discussion}

\subsection{Results using different setups}

In Table 2, the results of the experiments with single, bagged and boosted Tilde decision trees (Blockeel and Raedt 1998) from ten-fold cross-validation can be found. In the first row, the baseline accuracy of $54.7 \%$ indicates that the models clearly improve upon random guessing. The second row shows the reference result by Middendorf et al. (2004), where alternating decision trees (ADTrees) (Freund and Mason 1999) were applied to a propositional version of the basic data (without the additional predicates). ADTrees are a machine learning technique combining boosting and decision trees, aiming at rule sets that are small and easy to interpret. ${ }^{10}$

\footnotetext{
${ }^{10}$ Another experiment with ADTrees (results shown in rows three and four of the table) is discussed below.
} 
Table 2 Experimental results from ten-fold cross-validation: input data, model, average runtime per fold (in seconds), predictive accuracy in \% (standard deviation in \%), sensitivity, specificity, area under ROC curve (AUC), and the number of nodes in tree(s). Different variants of the data are tested: all includes relations gene, hasTFBS, isExpressed, and assignedToFuncat. -FunCat includes relations gene, hasTFBS, and isExpressed. -TFBS includes relations gene, isExpressed, and assignedToFuncat. $+P P I$ includes relations gene, hasTFBS, isExpressed, assignedToFuncat, and hasPPI restricted to manually annotated PPIs. ADTs $^{1}$ denotes the ADTree implementation in the WEKA workbench (Witten and Frank 2005) running over 10 iterations, $\mathrm{ADTs}^{2}$ denotes this implementation running over 100 iterations

\begin{tabular}{|c|c|c|c|c|c|c|c|}
\hline Data & Model & Time & Acc. & Sens. & Spec. & AUC & Size \\
\hline all & baseline & - & 54.7 & 0.0 & 100.0 & 50.0 & 0 \\
\hline -FunCat & ADTs (Middendorf et al. 2004) & - & 88.5 & - & - & - & - \\
\hline -FunCat & $\mathrm{ADTs}^{1}$ & 191 & $70.3(0.4)$ & 63.8 & 75.5 & - & 31 \\
\hline -FunCat & $\mathrm{ADTs}^{2}$ & 8,723 & $81.9(0.3)$ & 76.9 & 84.5 & - & 301 \\
\hline all & single Tilde & 185 & $85.1(0.9)$ & 87.1 & 83.5 & 92.8 & 186 \\
\hline all & bagging & 951 & $85.1(0.6)$ & 87.3 & 83.4 & 93.0 & 1,202 \\
\hline -FunCat & bagging & 921 & $80.6(0.7)$ & 78.6 & 82.4 & 88.7 & 1,388 \\
\hline$-T F B S s$ & bagging & 1,113 & $78.3(1.1)$ & 85.1 & 72.6 & 87.4 & 906 \\
\hline all & boost. & 2,658 & $91.2(0.5)$ & 91.2 & 91.3 & 97.7 & 2,491 \\
\hline -FunCat & boost. & 2,052 & $88.4(0.2)$ & 87.6 & 89.1 & 96.3 & 2,620 \\
\hline$-T F B S s$ & boost. & 1,902 & $83.4(0.9)$ & 87.7 & 77.7 & 90.9 & 1,774 \\
\hline$+P P I$ & boost. & 3,292 & $91.0(0.3)$ & 91.0 & 90.9 & 97.7 & 2,484 \\
\hline -FunCat & $\mathrm{ADTs}^{1}$ rand. & 167 & $59.1(0.2)$ & 44.5 & 71.1 & - & 31 \\
\hline -FunCat & $\mathrm{ADTs}^{2}$ rand. & 6,681 & $71.6(1.1)$ & 66.6 & 75.6 & - & 301 \\
\hline all & single rand. & 153 & $60.4(0.7)$ & 55.8 & 64.2 & 64.7 & 249 \\
\hline all & bagg. rand. & 1,116 & $63.3(0.1)$ & 47.6 & 76.3 & 68.2 & 1,634 \\
\hline -FunCat & bagg. rand. & 837 & $62.8(0.2)$ & 43.8 & 78.6 & 67.0 & 1,628 \\
\hline$-T F B S s$ & bagg. rand. & 1,148 & $61.6(1.1)$ & 37.1 & 81.9 & 63.7 & 863 \\
\hline all & boost. rand. & 1,909 & $67.5(0.3)$ & 62.1 & 72.0 & 76.3 & 3,005 \\
\hline -FunCat & boost. rand. & 1,491 & $66.8(1.1)$ & 60.5 & 72.0 & 76.0 & 2,961 \\
\hline$-T F B S s$ & boost. rand. & 1,830 & $62.2(0.7)$ & 49.2 & 73.0 & 66.3 & 2,311 \\
\hline$+P P I$ & boost. rand. & 2,305 & $64.9(0.1)$ & 59.4 & 69.5 & 72.0 & 2,965 \\
\hline all & single regr. & - & 83.2 & 84.4 & 82.4 & - & - \\
\hline
\end{tabular}

We included the results both with and without the use of FunCat terms, as well as with and without the use of transcription factor binding site data. In this way, we can estimate the relative contribution of different types of data to the prediction performance. In experiments including all protein-protein interactions (i.e., also data from high-throughput experiments), those predicates were not frequently used in the trees (results not shown in table). However, experiments with only manually annotated PPIs improved the results slightly (see below). Given the same information as Middendorf et al., i.e., given isExpressed, hasTFBS and gene, boosted decision trees are on par with ADTrees, whereas FunCat terms substantially improve the performance, both in terms of predictive accuracy and compactness.

Leaving transcription factor binding site information out results in a more compact model compared to the two other variants, at the expense of an increased imbalance between sensitivity and specificity, in favor of sensitivity. Predictive accuracy also drops substantially, from $91.2 \%$ to $83.4 \%$. The imbalance between specificity and sensitivity is much less pro- 
nounced in the setup where FunCat data was left out. Here, both measures are reduced almost equally.

As we can see, FunCat data as well as binding site information are beneficial for the learning task. The behavior of the regulators (relation isExpressed) in isolation (without any further information) turned out to be on the level of random guessing, i.e., not sufficient for gene regulation prediction. Nevertheless, the isExpressed predicate is needed because it characterizes the experimental conditions and because the behavior of regulators under a specific condition is biologically important for gene regulation.

In the following, we will discuss aspects of relational and propositional learning in the context of this dataset. First, it should be noted that the dataset (without PPIs) can be made propositional in several ways. The most important information for the prediction task is the presence of $m$ transcription factor binding sites and the state of $n$ regulators. Therefore, we could present feature vectors of size $m+n$ to the learning algorithms. This representation would be unnecessarily redundant (i.e., the state information of regulators would be replicated $m$ times) and large, but the output would be the same as obtained directly on the relational representation. In this way, however, the fact that binding sites and regulators are strongly connected is lost, making it hard for learning algorithms to detect information useful for classification. Another possible propositional representation avoiding this problem contains feature vectors of size $m \times n$, that is, one feature for each combination of binding site and regulator. This is the representation tested in the original ADTree experiment by Middendorf et al. To compare these representations, we conducted experiments with ADTrees applied to the simpler $m+n$ representation (see Table 2, results with 10 iterations in row 3 and 100 iterations in row 4). While the effort is much smaller compared to the $m \times n$ representation, the performance also drops significantly compared to the more complex representation. One of the useful features of relational learning algorithms is the possibility to fine-tune the language bias declaratively. Since we did not want to multiply the number of features in our representation (as in the original ADTree experiments), we used the declarative bias of Tilde to make sure that a test for information dependent on the experimental condition is only introduced once the state of a regulator is tested. In this way, we keep the space requirements of the $m+n$ representation and achieve about the same effect as in the $m \times n$ representation. This highlights a nice property of relational learning systems: the flexibility to adjust their bias to complex datasets.

In another experiment, we removed uncertain PPI data from high-throughput experiments to keep only strongly confirmed protein-protein interactions. Unfortunately, only a very small number of PPIs is left for regulators, rendering the approach useless for this part of the data. However, for the genes to be predicted, keeping only confirmed PPIs gives a result not significantly worse than the best overall result. Also, the difference between original and randomized data is maximal (see below), while the standard deviation is reduced. In this sense, the truly relational PPI information helps improving the performance even further.

To test the reliability of the results, we performed experiments with randomized versions of the original data. To do so, we randomly shuffled the values in each column of each relation except gene. As can be seen in the lower half of Table 2, the difference between original and randomized data ranges between 10 and 26 points. In particular, the difference between the best results using boosting is 26.1 , which is also among the biggest differences observed over a wider range of experiments (Fröhler 2006).

The standard deviations from ten-fold cross-validation (see Table 2) as well as the individual results of each run (not shown) suggest that the differences in performance cannot be attributed to random variation: Due to the size of the dataset, the standard deviations are relatively small, with only little overlap in the range above or below the mean cross-validation 
accuracy. It is also the case that for almost all pairs of methods, the performance difference is consistent over all cross-validation sets.

Next, we tested the value of the discretization. A Tilde regression tree was induced on the undiscretized numeric values greater than 1.2 and less than -1.2 (values in between are considered noisy and unreliable). The resulting model was used to classify instances: If the sign of the regression prediction is greater than zero, then the model predicts the positive class, otherwise, it predicts the negative class. In the last line of Table 2, we see that the results are not encouraging: The predictive accuracy of a single regression tree is about two points worse than the one of a single decision tree.

To check the dependence of the results on the ILP system chosen, we also conducted experiments with Aleph, a generic framework supporting mostly Progol-type learning. Due to the very large search space of clauses subsuming the bottom-clause, stochastic local search variants are the only option. Given the relatively large dataset of tens of thousands of examples, it turned out to be extremely hard to find a well-balanced set of clauses with suitable coverage and generalization performance. In fact, we did not manage to obtain results that are competitive with those presented here within reasonable time.

Finally, using a different set of transcription factors due to Pilpel (Fröhler 2006), it is possible to improve the results further to $92.8 \%$ in ten-fold cross-validation (results not shown in the table).

In summary, we presented experimental results of Tilde and ADTrees on various representations of the data. The first main advantage of relational learning and ILP in this domain seems to be the flexibility in defining the language bias, e.g., allowing only certain combinations of variables, or allowing certain variables to enter the model only once other variables are already included. The second main advantage is the intrinsic extensibility, for instance, the possibility to integrate functional categorizations and protein-protein interaction data.

\subsection{Extracting important features}

We will now inspect the learned model from the boosting setup using all information and extract biologically important features. We used two different ranking criteria to determine biologically relevant features. Firstly, we rank the features used in the model by a feature abundance score, meaning that features occurring in many branches are important for the prediction process of many different groups of genes. Secondly, we rank the features by a feature coverage score, counting the number of examples that are classified by a specific feature. Important features will classify a large number of examples with high accuracy. The scoring functions are not standard measures, but defined in analogy to those of Middendorf et al. We will now discuss the top 5 ranking features of each type of information.

\subsubsection{Important transcription factor binding sites}

The top-scoring binding site based on the abundance score as well as the coverage score is the MIG1 binding site which has been shown to be important for glucose metabolism and during carbon source based stress. Another top-scoring binding site is the MSN2/MSN4 binding site which is known to be a regulatory element for a significant number of general stress response target genes. Additional top-scoring binding sites are: GCN4 (amino acid biosynthesis and starvation response), TBP (TATA binding site), ADR1 (glycerol metabolism and osmolarity) and GAL4 (galactose metabolism). The names of the binding site refer to the transcription factor that is known to bind to that site. 


\subsubsection{Important transcription factors}

The top-scoring transcription factor based on the abundance score is USV1, which has also been found to be top-ranking by Segal et al. (2003). USV1 has been shown to be overexpressed during starvation. In the coverage-score ranking, PPT1 is the top-scoring regulator. PPT1 is a phosphatase which is expressed during logarithmic growth. In our model, the under-expression of PPT1 is the top-scoring feature based on the coverage score, which might be because most of the experimental conditions studied here are different from conditions where the cell undergoes logarithmic growth. The following transcription factors are ranked three to five in both rankings: SIP4 (transcriptional activator, binds to carbon sourceresponsive element (CSRE), involved in the positive regulation of gluconeogenesis), BMH1 (signaling molecule, interacts with TPK $1^{11}$ ) and TOS8 (regulates expression of genes involved in G1/S phase events).

\subsubsection{Important functional categories}

The top-scoring functional category based on the abundance score is FunCat 12.01 (ribosome biogenesis), whereas based on the coverage score, the more general FunCat 12 (protein synthesis) can be found to be top-scoring. Vice versa, FunCat 12.01 ranks second in the coverage score, and FunCat 12 ranks second in the abundance score. The third-top-scoring category in both rankings is 11.04.01 (rRNA processing). These three categories are important for protein synthesis and transcription in general and ribosome biogenesis in particular. Other top-scoring categories are: 01 (metabolism), 32.01 (stress response) and 02 (energy).

Since Gasch et al. (2000) expected the most significant changes in the stress response conditions studied involving protein synthesis, and particularly ribosome biogenesis and RNA metabolism, as well as cell growth, the features identified by our method are in accordance with biological expectations.

\subsection{Relating the features to the experimental conditions}

In the previous section, we inspected important individual features discovered by our model. We will now inspect which combinations of these features are used by our model and relate these combinations to the experimental conditions.

Since the synthesis of ribosomes consumes a vast amount of resources in rapidly growing cells, this process is known to be a key control point for regulation of cell growth and division in yeast. This synthesis pathway requires a large number of trans-acting factors and the depletion of cytoplasmic ribosomes leads to the expected phenotype: G1/S arrest. Because of this, ribosome synthesis is known to be closely integrated with cell growth and division (Dez and Tollervey 2004).

The first feature we want to inspect is the protein kinase PPT1, which is associated to cell growth and controlled by metabolic/nutritional signals. PPT1 is primarily controlled at the level of transcription (Jeong et al. 2003). This kinase (in state -1) can be found together with the functional categories 12 (protein synthesis), 12.01 (ribosome biogenesis), 11.02.01 (rRNA synthesis), 11.04.01 (rRNA processing). Genes covered by the respective rules are predicted to be under-expressed (state -1 ). Knowing about the close integration of cell cycle

\footnotetext{
${ }^{11}$ TPK1 post-translationally regulates the transcription factor MSN2 which in turn can bind to the MSN2/MSN4 transcription factor binding site (stress response element).
} 
and ribosome biogenesis, these rules can be interpreted in the light of biological knowledge. PPT1 was also predicted to be associated to the cell cycle by Segal et al. (2003).

PPT1 (in state -1) can also be found together with the MSN2/MSN4 binding site (environmental stress response element) plus two instances of the MIG1 binding site. Genes covered by the respective rule are predicted to be over-expressed. Whereas genes containing the MSN2/MSN4 and the MIG1 binding site are predicted to be over-expressed, genes lacking the MSN2/MSN4 binding site (and only containing the MIG1 site) are predicted to be under-expressed. Since the MSN2/MSN4 binding site represents the environmental stress response element, these findings also appear consistent with biological knowledge. PPT1 (again in state -1 ) additionally can be found together with the binding site for the heat shock factor HSF1, which is an activator and essential for growth. HSF1 mediates expression of heat-shock genes in response to elevated temperatures (Park and Craig 1989; Wiederrecht et al. 1988).

The second feature we want to inspect is USV1, whose biological function currently is unknown (www.yeastgenome.org 2006). USV1 appears to be up-regulated during starvation and can be found (in state +1 ) together with the functional categories 12.01 (ribosome biogenesis) and 11.04.01 (rRNA processing). Genes covered by these rules are predicted to be under-expressed. USV1 (in state +1 ) can also be found together with the INO2/INO4 binding site (activator, required for derepression of phospholipid biosynthetic genes (www.yeastgenome.org 2006)). Genes covered by that rule are predicted to be overexpressed. So, our model hypothesizes that USV1 is involved in ribosome synthesis and phospholipid biosynthesis.

The regulators SIP4 and BMH1 both can be found independently of each other together with the functional category 12.01 (ribosome biogenesis), in the case of SIP4 with an additional MIG1 binding site. The up-regulation of SIP4 and BMH1 respectively together with the FunCat 12.01 leads to a predicted down-regulation of the genes covered by the respective two rule. Whereas SIP4 is a transcription factor, BMH1 is a signaling molecule. Additionally, SIP4 together with the regulator REG2 (both in state -1) leads to a predicted under-expression of genes under the respective conditions. REG2 is a phosphatase which regulates SNF1 (a kinase) which in turn activates SIP4. SIP4 finally binds to the carbon source responsive element (CSRE) (www.yeastgenome.org 2006). BMH1 can also be found together with the MSN2/MSN4 binding site plus two MIG1 binding sites. Since the signaling molecule BMH1 interacts with TPK1 which in turn controls the MSN2/MSN4 level in the nucleus (www.yeastgenome.org 2006), this association appears consistent with known biology. Also, the MSN4 transcription factor itself can be found together with its MSN2/MSN4 binding site plus two MIG1 binding sites.

In summary, we found many different ways of relating important features of our model to the biological literature. In particular, it should be noted that it was not possible to detect a relationship between transcription factors and FunCat terms in the previous approach using ADTrees.

\section{Conclusion and discussion}

We presented a new application of ILP to gene regulation prediction. The results demonstrate that it is possible to derive a strong predictive model for this task. Methods from Inductive Logic Programming are particularly useful, since the bias can be adjusted to complex datasets, and information can be integrated and tested easily. Moreover, informative features can be extracted from boosted first-order decision trees in a style similar to 
random forests (Breiman 2001): The co-occurrence of features may hint at relevant combinations of binding sites and regulators, and can be interpreted in the light of biological literature. The data described in this paper are made available on our web site (see http://wwwkramer.in.tum.de/research).

Interestingly, this work is related to a paper by Ong et al. (2006). Both approaches aim at the modeling of gene regulation in the framework of ILP. The main difference is that we do not explicitly build a dynamic model of the time course of gene expression. The approach of Ong et al. uses time series information from the Gasch dataset and learns recursive clauses modeling the change of gene expression over time. By contrast, our approach appears more comprehensive, but also less ambitious, as only a static model of gene regulation is learned from the complete Gasch data.

Although we tested our model on a eukaryotic, and not on a prokaryotic organism, it is clear that regulation in higher eukaryotes is much more complicated and at present not fully understood. Still, it is a valid approach to use all available information on a simpler model organism and build a model of an important biological aspect as accurately as possible. It is an open question whether the methods presented in the paper are scalable to more complex species.

Various other sources of information could be integrated to improve the performance of the model. Obviously, pathway information could be taken into account. However, it may be argued that the same information is already available in the form of functional categorizations. In future work, we are planning to refine our approach as follows. First, it would make sense to differentiate between binding sites according to their position in the regulatory region. Second, the use of phylogenetic information should improve performance even further. Vice versa, predictive models of gene regulation for various organisms could provide interesting information for phylogenies.

Acknowledgements We would like to thank Thomas Werner and Bernhard Pfahringer for valuable comments on an earlier draft of this paper.

\section{References}

Allocco, D. J., Kohane, I. S., \& Butte, A. J. (2004). Quantifying the relationship between co-expression and co-regulation and gene function. BMC Bioinformatics, 5(18)

Blockeel, H., \& Raedt, L. D. (1998). Top-down induction of first-order logical decision trees. Artificial Intelligence, 101(1-2), 285-297.

Breiman, L. (2001). Random forests. Machine Learning, 45(1), 5-32.

Dez, C., \& Tollervey, D. (2004). Ribosome synthesis meets the cell cycle. Current Opinion in Microbiology, 7(6), 631-637.

Freund, Y., \& Mason, L. (1999). The alternating decision tree learning algorithm. In Proceedings 16th international conference on machine learning (ICML 1999) (pp. 124-133). Los Altos: Kaufmann.

Fröhler, S. (2006). Machine learning for gene regulation prediction. Diploma thesis, TU München.

Gasch, A. P., Spellman, P. T., Kao, C. M., Carmel-Harel, O., Eisen, M. B., Storz, G., Botstein, D., \& Brown, P. O. (2000). Genomic expression programs in the response of yeast cells to environmental changes. Molecular Biology of the Cell, 11(12), 4241-4257.

Jeong, J., Johns, J., Sinclair, C., Park, J., \& Rossie, S. (2003). Characterization of Saccharomyces cerevisiae protein Ser/Thr phosphatase T1 and comparison to its mammalian homolog PP5. BMC Cell Biology, 4(3).

Latchman, D. (2005). Gene regulation: a eukaryotic perspective (5th ed.). London: Taylor \& Francis.

Mewes, H., Albermann, K., Heumann, K., Liebl, S., \& Pfeiffer, F. (1997). MIPS: a database for protein sequences, homology data and yeast genome information. Nucleic Acids Research, 25(1), 28-30.

Middendorf, M., Kundaje, A., Wiggins, C., Freund, Y., \& Leslie, C. (2004). Predicting genetic regulatory response using classification. Bioinformatics, 20(suppl_1), 232-240. 
Ong, I., Page, D., \& Santos Costa, V. (2006). Inferring regulatory networks from time series expression data and relational data via inductive logic programming. In Proceedings 16th International Conference on Inductive Logic Programming (ILP 2006), short papers.

Park, H.-O., \& Craig, E. A. (1989). Positive and negative regulation of basal expression of a Yeast HSP70 gene. Molecular and Cellular Biology, 9(5), 2025-2033.

Ruepp, A., Zollner, A., Maier, D., Albermann, K., Hani, J., Mokrejs, M., Tetko, I., Guldener, U., Mannhaupt, G., Munsterkotter, M., \& Mewes, H. W. (2004). The FunCat, a functional annotation scheme for systematic classification of proteins from whole genomes. Nucleic Acids Research, 32(18), 5539-5545.

Segal, E., Shapira, M., Regev, A., Pe'er, D., Botstein, D., Koller, D., \& Friedman, N. (2003). Module networks: identifying regulatory modules and their condition-specific regulators from gene expression data. Nature Reviews Genetics, 34(2), 166-167.

van Helden, J., Andr, B., \& Collado-Vides, J. (1998). Extracting regulatory sites from the upstream region of yeast genes by computational analysis of oligonucleotide frequencies. Journal of Molecular Biology, $281,827-842$.

Wiederrecht, G., Seto, D., \& Parker, C. (1988). Isolation of the gene encoding the S. cerevisiae heat shock transcription factor. Cell, 54(6), 841-853.

Wingender, E., Dietze, P., Karas, H., \& Knueppel, R. (1996). TRANSFAC: a database on transcription factors and their DNA binding sites. Nucleic Acids Research, 24(1), 238-241.

Witten, I. H., \& Frank, E. (2005). Data mining: practical machine learning tools and techniques (2nd ed.). Los Altos: Kaufmann.

www.yeastgenome.org 